\title{
Perfil dos participantes do programa permanente de prevenção e diagnóstico precoce das doenças bucais, com ênfase no câncer de boca, no município de Cuiabá-MT
}

\author{
Participants profile of the permanent program of prevention and early diagnosis \\ of oral diseases, with emphasis on oral cancer, in the city of Cuiabá-MT
}

\section{Perfil de los participantes en el programa permanente de prevención y diagnóstico precoz de enfermedades orales, con énfasis en el cáncer oral en la ciudad de Cuiabá-MT}

\author{
Bruna Lorrany Rodrigues da SILVA ${ }^{1}$ \\ Manoel dos Santos da SILVA NETO ${ }^{1}$ \\ Diurianne Caroline Campos FRANÇA ${ }^{2}$ \\ Sandra Maria Herondina Coelho Ávila de AGUIAR ${ }^{3}$ \\ ${ }^{1}$ Graduando(a) em Odontologia pelo Centro Universitário de Várzea Grande, UNIVAG, \\ 78118-900 Várzea Grande - MT, Brasil \\ ${ }^{2}$ Professora do Curso de Odontologia, Centro Universitário de Várzea Grande, UNIVAG, \\ 78118-900 Várzea Grande - MT, Brasil \\ ${ }^{3}$ Professora Adjunto, Faculdade de Odontologia de Araçatuba \\ Universidade Estadual Paulista, UNESP, 16015-050, Araçatuba - SP, Brasil
}

\begin{abstract}
Resumo
O objetivo deste estudo foi conhecer o perfil dos participantes do programa permanente de prevenção e diagnóstico precoce das doenças bucais, com ênfase no câncer de boca, no município de Cuiabá, de agosto de 2014 a julho de 2015. Foram analisadas 600 fichas individuais dos pacientes atendidos neste programa permanente, cujos dados referentes às variáveis idade, sexo, cor da pele, hábitos, doenças pré existentes e variações de normalidade foram tabulados. Houve maior predomínio de pacientes do sexo masculino $(53,5 \%)$ e com faixa etária de maior participação dos 20-29 (27,83\%). As doenças pré-existentes relatadas somaram 138 pessoas. Foram encontradas 1607 variações da normalidade. Concluiu-se que as campanhas para a busca ativa das lesões precursoras do câncer bucal são importantes e devem ser estendidas a toda população de Cuiabá, tanto pelas instituições de ensino, como também por outras entidades e instituições de saúde.

Descritores: Diagnóstico Bucal; Prevenção Primária; Epidemiologia.
\end{abstract}

\begin{abstract}
The objective of this study was to know the participants profile of the permanent program of prevention and early diagnosis of oral diseases, emphasizing oral cancer in the city of Cuiabá, from August 2014 to July 2015. Six hundred patient records were analysed, whose data regarding age, sex, skin color, habits, pre-existing diseases and normality variations were tabulated. There was a higher prevalence of male patients (53.5\%) and the age group with 20-29 $(27.83 \%)$. Pre-existing diseases reported totaled 138 people. There were 1607 variations of normality. It was concluded that campaigns for the active search for oral cancer precursor lesions are important and should be extended to all Cuiabá population, by educational institutions, as well as by health institutions and others institutions.

Descriptors: Diagnosis, Oral; Primary Prevention; Epidemiology.
\end{abstract}

\begin{abstract}
Resumen
El objetivo de este estudio fue conocer el perfil de los participantes en el programa permanente de prevención y diagnóstico precoz de enfermedades orales, con énfasis en el cáncer oral en la ciudad de Cuiabá, a partir de agosto de 2014 y julio de 2015. Se analizaron 600 registros de pacientes individuales cumplido este programa en curso, cuyos datos sobre la edad, el sexo, el color de la piel, los hábitos, las enfermedades preexistentes y los cambios normales fueron tabulados. Hubo una mayor prevalencia de pacientes del sexo masculino $(53,5 \%)$ y la edad mayor participación de 20-29 (27,83\%). Enfermedades preexistentes reportadas ascendieron a 138 personas. Se encontraron 1607 variaciones de la normalidad. Se concluyó que las campañas para la búsqueda activa de lesiones precursoras de cáncer oral son importantes y deben extenderse a toda la población de Cuiabá, tanto por las instituciones educativas, así como por otras entidades e instituciones de salud.

Descriptores: Diagnóstico Bucal; Prevención Primaria; Epidemiología.
\end{abstract}

\section{INTRODUÇÃO}

O câncer vem ocupando papel cada vez mais importante no perfil de mortalidade do Brasil. Dentre os tipos mais prevalentes, o câncer bucal aparece em oitavo lugar, com incidência e mortalidade crescentes. Somando-se à mortalidade, a morbidade associada ao câncer bucal tem impacto importante na qualidade de vida dos pacientes, pois seu diagnóstico tem sido realizado tardiamente, reduzindo as possibilidades de cura e aumentando as mutilações decorrentes do tratamento. A participação do cirurgião dentista nas ações de prevenção e diagnóstico precoce do câncer bucal é indispensável, pois são os profissionais mais capacitados a informar seus pacientes e a população sobre o câncer bucal, devido a sua área de atuação ser especificamente as regiões onde ocorre a neoplasia. Portanto, estratégias de detecção precoce do câncer bucal reduzem sua incidência e mortalidade ${ }^{1}$. 
Contrário a outros tipos de câncer, que requerem ferramentas mais sofisticadas para o diagnóstico, o câncer bucal é facilmente detectado em um exame clínico de rotina, pois na maioria das vezes é precedido por lesões prémalignas. Sendo assim, o retardo no diagnóstico se deve não só ao desinteresse dos pacientes em procurar ajuda profissional, mas a falta de sensibilidade dos cirurgiões dentistas em relação a atitudes de prevenção ${ }^{2}$.

A prevenção primária, os diagnósticos clínicos e histopatológicos precoces e a reabilitação maxilofacial estão no quadro de obrigações da odontologia e cada cirurgião dentista deve conhecer o assunto, suas possibilidades de atuação e os limites legais entre as profissões que atuam no tratamento do câncer bucal ${ }^{3}$.

No Brasil, percebe-se que a realidade socioeconômica também interfere no quadro da doença, dada a maior incidência em pessoas de baixa renda, carentes de recursos e também de informações. Uma população que não tem o hábito de cuidar de si, não percebe as lesões precursoras do câncer bucal e, finalmente, com dificuldade de acesso aos serviços de saúde ${ }^{4}$.

A identificação de lesões bucais é fundamental na prática profissional, assim como o reconhecimento dos aspectos clínicos das doenças e a probabilidade das mesmas ocorrerem, de acordo com as características de um determinado grupo de indivíduos. O Instituto Nacional de Câncer estima que em 2015, no Brasil, haverá 9990 novos casos de homens e 4180 novos casos de mulheres com neoplasia maligna da cavidade oral. Em Mato Grosso, a estimativa segundo o sexo, é de 120 novos casos em homens e $40 \mathrm{em}$ mulheres considerando as taxas brutas de incidência por 100 mil habitantes Esses dados apontam para a necessidade de que os profissionais estejam aptos a realizar um exame clínico detalhado e minucioso, e consequentemente um diagnóstico correto ${ }^{5}$.

Essa enfermidade tem comportamento agressivo, o tratamento é mutilante, além de ser excludente social. A taxa de mortalidade da doença está em torno de 50 por cento dos casos, constituindo assim um problema de saúde pública. Considerando o elevado índice de morbidade e mortalidade e pela grande possibilidade de diminuição destes índices através da identificação precoce, os esforços do sistema de saúde em relação a esse problema deve se concentrar na educação do público, informando-o quanto aos fatores de risco, sinais e sintomas de alerta, bem como sobre a realização do autoexame, e capacitação dos profissionais Cirurgiões Dentistas, que tem contato frequente com a cavidade bucal em sua atividade profissional. Cabe a estes profissionais realizarem exame clinico minucioso e detalhado, afastar os fatores carcinogênicos, reconhecer e tratar as lesões cancerizáveis, bem como ter conhecimento dos exames complementares para o diagnóstico das neoplasias malignas.

Desta maneira tem-se a oportunidade do diagnóstico precoce do câncer de boca, detectando-o em estágios incipientes.

O problema do câncer no Brasil ganha relevância pelo perfil epidemiológico que essa doença vem apresentando, e, com isso, o tema tem conquistado espaço nas agendas políticas e técnicas de todas as esferas de governo.

Conforme Vidal et al. ${ }^{6}$ o alto índice de mortalidade por câncer bucal é inadmissível quando a prevenção e o diagnóstico precoce podem ser promovidos, bastando para isto interesse e capacitação adequada.

Levando em consideração os aspectos abordados, e que a literatura é escassa sobre este assunto, justificou-se a realização desta pesquisa analisando os dados do programa permanente de prevenção e diagnóstico precoce das doenças bucais com ênfase no câncer de boca, realizado no município de Cuiabá, entre os meses de agosto de 2014 a julho de 2015, onde o objetivo foi de verificar o perfil dos participantes das campanhas realizadas bem como de rastear as lesões em fases iniciais e sensibilizar a população quanto à importância da realização do autoexame e sobre os fatores de risco das doenças bucais.

\section{MATERIAL E MÉTODO}

O estudo realizado se caracterizou por ser uma pesquisa quantitativa, descritiva e exploratória, de corte transversal, servindo-se de dados secundários de 600 fichas dos participantes, obtidos durante as 09 campanhas de diagnóstico realizadas entre agosto de 2014 a julho de 2015.

Para coleta de dados foram utilizadas as fichas dos pacientes examinados, preenchidas pelos acadêmicos participantes do programa. Os mesmos foram capacitados com 20 horas de aulas teóricas e 8 horas de aulas práticas, abordando um detalhado e minucioso exame clínico, com a verificação de variações de normalidade ou alterações dos tecidos do sistema estomatognático, realizando o diagnóstico quando necessário através de exames complementares como radiografias, biópsias e citologias esfoliativas. Os alunos envolvidos faziam o exame clínico e na sequência chamavam o professor responsável para finalizar o atendimento, além de propor diagnóstico e tratamento caso houvesse necessidade.

Durante o atendimento foi realizado exame clínico, constituído por anamnese, na qual se fez o preenchimento da ficha clínica e a entrevista do participante. Posteriormente realizou-se o exame físico que constou do exame extra e intrabucal (exame detalhado e minucioso das estruturas bucais, com a realização da palpação das estruturas bucais e pescoço, seguindo o que preconiza a técnica semiológica recomendada pela American Cancer Society). Foram utilizados instrumentais e equipamentos odontológicos para os exames, tais como caixas para exame clinico, fonte de luz direta na cadeira odontológica, equipamentos de proteção individual e descartáveis, espátulas de madeira, compressas de gazes estéreis. Nos casos que tiveram indicação de coleta de material através de citologia e/ou biópsia foi feito encaminhamento para os Centros de Especialidades Odontológicas (CEO's) e para a Clínica de Odontologia do UNIVAG, onde as coletas foram feitas com materiais padronizados e enviados para exames citopatológico e histopatológico para elucidar o diagnóstico. Os casos confirmados de câncer de boca foram encaminhados para os centros de referência para realizar o tratamento. Os demais pacientes que não apresentarem lesões de boca, mas tiveram necessidade de tratamento odontológico (curativo) foram encaminhados para agendamento nas respectivas clínicas.

As informações das fichas foram transcritas por um único pesquisador em tabela elaborada para esta pesquisa. Os dados analisados foram: sexo, faixa etária, cor da pele, estado civil, hábitos, doenças pré-existentes e variações da normalidade.

As idades foram agrupadas nas seguintes faixas etárias: 0 a 9 anos, 10 a 19 anos, 20 a 29 anos, 30 a 39 anos, 40 a 49 anos, 50 a 59 anos, 60 a 69 anos, maiores de 70 anos. No que se refere ao sexo do participante, foram definidos em masculino e feminino; a etnia/raça foi dividida em melanoderma, feoderma, leucoderma e xantoderma. $\mathrm{O}$ estado civil foi registrado como solteiro, casado, viúvo, divorciado, união estável e outros. 
Os hábitos avaliados foram etilismo, tabagismo, etilismo e tabagismo (ambos) e outros. As doenças préexistentes relatadas nas fichas individuais dos participantes foram transcritas e classificadas em: anomalias congênitas, transtornos psiquiátricos, distúrbios sensoriais, doenças sistêmicas crônicas, doenças infectocontagiosas, condições sistêmicas e outros, segundo classificação de SabbaghHaddad e Magalhães ${ }^{7}$.

$\mathrm{Na}$ análise de variações da normalidade foram consideradas todas as alterações de estruturas anatômicas que estivessem fora do padrão da normalidade bucal, porém, que não caracterizasse qualquer patologia, segundo classificação de Neville ${ }^{8}$.

Importante ressaltar que cárie e doença periodontal são condições patológicas que foram desconsideradas nessa classificação, pois não caracterizaram objeto deste estudo.

Os resultados foram analisados estatisticamente utilizando tabelas do Microsoft Office Word e Microsoft Office Excel, versão 2010, visando facilitar a identificação e a análise dos dados relevantes para este estudo.

\section{RESULTADOS}

Após nove campanhas realizadas nos períodos de agosto de 2014 a julho de 2015, das 600 pessoas participantes, 53,5\% eram do sexo masculino. A faixa etária de maior prevalência foi dos 20-29 anos $(27,83 \%)$ seguido de 30-49 anos (19,6\%). Quanto a cor da pele, $49 \%$ das pessoas se consideraram melanodermas, $27,5 \%$ leucodermas, $15,8 \%$ feodermas, $4,6 \%$ xantodermas e $3 \%$ não constavam essa informação.

Dentre os hábitos/vícios relatados pelos participantes, 383 pessoas $(63,8 \%)$ relataram não possuir nenhum hábito, $117(19,5 \%)$ declararam etilistas, 44 (7,3\%) tabagistas, 35 $(5,8 \%)$ relataram ser tabagista e etilista, $16(2,6 \%)$ são extabagistas e $06(0,8 \%)$ disseram possuir outros hábitos (Tabela 1).

No que se refere às doenças pré-existentes 138 (23\%) pessoas relataram alguma alteração, sendo que, as mais prevalentes foram as doenças do aparelho circulatório 88 pessoas $(63,7 \%)$ e $30(5 \%)$ com alterações endócrinas e 8 $(1,3 \%)$ casos do aparelho digestivo.

Das 600 fichas analisadas, observou-se 1.607 variações de normalidades, dentre elas as mais comuns foram língua saburrosa 293 (18,2\%), 240 casos de linha Alba (14,9\%), 177 (11\%) de pigmentação melânica racial,156 (9,7\%) grânulos de Fordyce, $133(8,2 \%)$ de papilas parotídea proeminente, $103(6,4 \%)$ de língua crenada (Tabela 2).

Os participantes que apresentaram qualquer lesão dos tecidos da boca foram encaminhados para esclarecimento diagnóstico através da realização de exames complementares.

No período estudado foram encaminhadas 135 pessoas para exames complementares, esclarecimento de diagnóstico e tratamento.

Tabela 1. Distribuição dos hábitos/vícios encontradas durante a anamnese dos participantes

\begin{tabular}{l|l|l}
\hline Hábitos & Quantidade & Porcentagem \\
\hline Etilista & 117 & $19,5 \%$ \\
\hline Tabagista & 44 & $7,33 \%$ \\
\hline Outros & 05 & $0,83 \%$ \\
\hline Não etilista e não tabagista & 383 & $63,83 \%$ \\
\hline Etilista e tabagista & 35 & $5,83 \%$ \\
\hline Ex tabagista & 16 & $2,66 \%$ \\
\hline
\end{tabular}

Tabela 2. Distribuição das variações de normalidade encontradas durante o exame físico intrabucal dos participantes

\begin{tabular}{l|l|l}
\hline Variações de normalidade & Quantidade & Porcentagem \\
\hline Grânulos de Fordyce & 156 & $9,70 \%$ \\
\hline Leucoedema & 23 & $1,43 \%$ \\
\hline Linha Alba & 240 & $14,93 \%$ \\
\hline Papila Parotídea Proeminente & 133 & $8,27 \%$ \\
\hline Pigmentação Melânica Racial & 177 & $11,01 \%$ \\
\hline Língua Crenada & 103 & $6,40 \%$ \\
\hline Língua Sulcada & 62 & $3,85 \%$ \\
\hline Língua Geográfica & 11 & $0,68 \%$ \\
\hline Língua Pilosa & 08 & $0,49 \%$ \\
\hline Língua Fissurada & 53 & $2,67 \%$ \\
\hline Língua Saburrosa & 293 & $18,23 \%$ \\
\hline Anquiloglossia & 27 & $1,68 \%$ \\
\hline Macroglossia & 04 & $0,24 \%$ \\
\hline Hiperplasia focal irritativa & 46 & $2,86 \%$ \\
\hline Papilas Linguais Hipertróficas & 37 & $2,30 \%$ \\
\hline Amígdala Lingual Hipertrófica & 12 & $0,74 \%$ \\
\hline Torus Palatino & 54 & $3,36 \%$ \\
\hline Torus Mandibular & 62 & $3,85 \%$ \\
\hline Varicosidade Lingual & 64 & $3,98 \%$ \\
\hline Fibrose do Ligamento Periodontal & 26 & $1,61 \%$ \\
\hline Fossetas Palatina de Stieda & 11 & $0,68 \%$ \\
\hline Exostose & 04 & $0,24 \%$ \\
\hline Anteriorização da Papila Incisiva & 01 & $0,06 \%$ \\
\hline
\end{tabular}

\section{DISCUSSÃO}

Segundo Hipólito e Martins ${ }^{9}$, estudos epidemiológicos constituem instrumento coletivo valioso na descrição das condições de saúde da população, na investigação de seus determinantes e nas ações destinadas a alterá-las.

Martins Filho ${ }^{10}$ assinala que os programas de controle do câncer são o instrumento mais eficaz para diminuir a distância entre conhecimentos e prática, permitindo que se alcance não só a redução na incidência da doença, como também da morbidade e mortalidade associadas, além da melhoria da qualidade de vida para pacientes e suas famílias.

A idade tem sido apontada como sendo um fator importante no aumento de lesões de mucosa bucal ${ }^{11}$. Contudo os participantes apresentaram idade relativamente jovem, mostrando um provável desinteresse da população adulta e idosa quando o assunto é saúde bucal. A faixa etária de maior prevalência foi dos 20-29 anos $(27,83 \%)$ seguido de 30-49 anos $(19,6 \%)$, esta última faixa etária mais prevalente está dentro do grupo alvo do programa.

A maioria dos participantes se considerou melanoderma (49\%), porém esses dados não condizem com as características raciais do município, segundo o último censo IBGE ${ }^{12}$

Sabe-se que o tabagismo e o álcool são fatores diretamente ligados a diversos tipos de cânceres, um exemplo é o elevado risco do desenvolvimento de câncer de boca e de lábios ${ }^{13}$. Das 600 pessoas atendidas, $117(19,5 \%)$ declararam ser etilistas, $44(7,33 \%)$ tabagistas e $35(5,83 \%)$ relataram ambos os hábitos.

Quanto às doenças sistêmicas, concordamos com os resultados de Melo et al. ${ }^{14}$ que também encontraram em estudo retrospectivo dos pacientes atendidos na clínica odontológica da Universidade de Vale do Rio Verde a hipertensão arterial sistêmica seguida pelo Diabetes, como doenças mais comuns. Na nossa pesquisa, essas duas patologias representaram 108 pessoas da nossa amostra Dessa forma sugere-se que o cirurgião-dentista esteja cada vez mais atento durante a anamnese e principalmente preparado para atender essa clientela com doenças sistêmicas, que a cada dia aumenta consideravelmente em nosso País.

As variações de normalidade encontradas assemelham-se com os dados encontrados por França et al. ${ }^{2}$, 
sendo que a variação mais encontrada foi a língua saburrosa $(18,23 \%)$, sugerindo uma deficiência de higiene bucal das pessoas, pois essa alteração está diretamente relacionada a falta de higienização da língua. Para reduzir esses dados, sugerem-se mais campanhas educativas e de prevenção, levando informações sobre os cuidados a população principalmente as comunidades de baixa renda.

Nossa pesquisa mostra o quão é importante o desenvolvimento de estratégias voltadas para prevenção e diagnóstico precoce, a fim de reduzir os problemas bucais. Tais estratégias devem ser estruturadas com uma visão mais ampla, voltadas para a redução não só de perdas dentárias, mas também para redução das demais patologias bucais.

\section{CONCLUSÃO}

O papel dos serviços de saúde, especialmente os do Sistema Único de Saúde (SUS), na prevenção e diagnóstico precoce das doenças da boca e da face são extremamente relevantes.

As doenças da boca, em particular o câncer bucal representa um importante problema de saúde pública no mundo, devido ao aumento da sua prevalência, além do grande impacto individual, institucional, social e econômico. Assim, merece uma abordagem ampliada, integral e interdisciplinar, a fim de minimizar as sequelas decorrentes da doença ou do seu tratamento, visando à cura com manutenção da qualidade de vida.

Portanto, os programas a serem instituídos, visando ações educativas, preventivas e curativas, bem como o planejamento em Odontologia, direcionados às doenças bucais deverão considerar as atitudes, valores, crenças, enfim, as representações sociais acerca das doenças bucais.

\section{REFERÊNCIAS}

1. Lima AAS, França BHS, Ignácio SA, Baione CS Conhecimento de alunos universitários sobre câncer bucal. Rev bras cancerol. 2005; 51(4):283-8.

2. França DCC, Duarte GC, Monteiro AD, Silva AALS, Aguiar SMHCA. Perfil epidemiológico dos participantes do Programa de diagnóstico e prevenção do câncer de boca em Mato Grosso.Arq Odontol. 2011; 47(2):90-4.

3. Almeida FCS, Cazal F, Brandão TB, Araújo ME, Silva DP, Dias RB. Campanha de popularização do autoexame da boca. Universidade de São Paulo, Brasil.(parte I). Rev bras patol oral. 2005; 4(3):147-56.

4. Matos IB, Araujo LA. Práticas acadêmicas, cirurgiões dentistas, população e câncer bucal. Revista da ABENO. 2003; 3(1):76-81.

5. Brasil. Ministério da Saúde. Secretaria de Atenção à Saúde. Instituto Nacional do Câncer. Coordenação de Prevenção e Vigilância. Estimativa 2010: Incidência de câncer no Brasil. Rio de Janeiro: INCA; 2015 [acesso em 2 de abril de 2015]. Disponível em: http://www.inca.gov. br/estimativa/2015/versaofinal.pdf.

6. Vidal AKL. Programa de combate ao câncer de boca. Odontol Clín-Cient. 2006; 5(1):63-8.

7. Sabbagh-Haddad A, Magalhães MGH. Odontologia para pacientes com necessidades especiais. São Paulo: Santos; 2007.

8. Neville BW. Patologia Oral e Maxilofacial. 2aed. Rio de Janeiro: Guanabara Koogan; 2004.

9. Hipolito RA, Martins CR. Prevalência de alterações da mucosa bucal em adolescentes brasileiros institucionalizados em dois centros de reeducação. Ciênc saúde coletiva. 2010; 15(supl 2):3233-42.
10. Martins Filho PRS, Santos TS, Silva LCF, Piva MR Prevenção e controle do câncer bucal no Brasil: uma história secular de Políticas Públicas de Saúde. RGO. 2014; 62(2):159-64.

11. Henrique PR, Furuse C, Junqueira JLC, Araújo VC, Bazaga Júnior M. Prevalência de alterações da mucosa bucal em indivíduos adultos da população de Uberaba, Minas Gerais. RGO. 2009; 57(3): 261-7.

12. Instituto Brasileiro de Geografia e Estatística (IBGE). Censo demográfico 2010. Características da população e dos municípios. Resultados do universo. Rio de Janeiro: IBGE, 2011. 270p.

13. Hendges DJB, Stoll RR, Moreschi C. A influência de hábitos e estilos de vida o surgimento de neoplasias malignas - uma revisão de literatura. Destaques Acadêmicos. 2013; 5(3):121-30.

14. Melo JC, Elias DC, Souza RD, Oliveira RL. Perfil do Pacientes Atendidos na Clínica Odontológica da UNINCOR. Revista UninCor. 2014; 12(1):614- 20.

\section{CONFLITO DE INTERESSES}

Os autores declaram não haver conflitos de interesse.

\section{AUTOR PARA CORRESPONDÊNCIA}

\section{Diurianne Caroline Campos França}

diurianne.odonto@terra.com.br

Submetido em 26/01/2017 Aceito em 16/02/2017 